\title{
QCD effective actions from the solutions of the transport equations
}

\author{
Cristina Manuel* \\ Theory Division, CERN, CH-1211 Geneva 23, Switzerland \\ Stanisław Mrówczyński ${ }^{\dagger}$ \\ Sottan Institute for Nuclear Studies, ul. Hoza 69, PL-00-681 Warsaw, Poland \\ and Institute of Physics, Świętokrzyska Academy, ul. Świętokrzyska 15, PL-25-406 Kielce, Poland
}

(Received 25 June 2002; published 21 January 2003)

\begin{abstract}
We solve the collisionless transport equations of a quark-gluon plasma interacting through mean chromodynamic fields. The system is assumed to be translation invariant in one or more space-time directions. We present exact solutions that hold if the vector gauge fields in the direction of the translation invariance commute with their covariant derivatives. We also solve the equations perturbatively when the commutation condition is relaxed. Further, we derive the color current and the associated effective action. For the static quasiequilibrium system, our results reproduce the full one-loop effective action of QCD in the presence of constant background fields, where the above mentioned commutation condition is satisfied.
\end{abstract}

DOI: $10.1103 /$ PhysRevD.67.014015

PACS number(s): 12.38.Mh, 05.20.Dd, 11.10.Wx

\section{INTRODUCTION}

When the temperature $T$ of the quark-gluon plasma is much greater than the QCD scale parameter $\Lambda_{\mathrm{QCD}}$, the hard modes, i.e. those with momenta of the order of $T$ or larger, are weakly interacting and they can be described within perturbative QCD [1]. The dynamics of the soft sector, however, remains nonperturbative even at arbitrarily large temperature [2], as signaled by severe infrared divergences [3]. Then, one has to refer to effective theories to get insight into the soft mode dynamics. Such theories, see e.g. [4,5], can be derived from QCD by integrating out the hard modes, but constructing them is by far not a simple task. Consequently, one often relies on more or less heuristic approaches, usually exploiting a semiclassical or classical field approximation because the occupation numbers of the soft gluonic modes are large.

A very natural effective approach is provided by the kinetic theory, where the hard modes are treated as (quasi)particles while the soft gluonic ones contribute to the chromodynamic mean field. The transport theory has been formulated in two versions. The first one treats the color degrees of freedom as a classical continuous variable which, as position or momentum, evolves in time. A starting point of the theory are the Wong equations [6], which describe a classical particle that interacts with the chromodynamic field due to the color charge. Then, one immediately gets the Liouville and the transport equations [7] of a many-body quark-gluon system. The physical content of the theory is rather transparent and numerous results, for example transport coefficients, can be easily obtained. Even the simplest collisionless transport equations, where the dissipation phenomena are neglected, provides a surprisingly rich dynamics. The transport theory with the classical color became really reliable when the theory was found [8] to reproduce the QCD hardthermal-loop dynamics $[4,9,10]$. It was further established

\footnotetext{
*Electronic address: cristina.manuel@cern.ch

†Electronic address: mrow@fuw.edu.pl
}

[11] that the theory supplemented by the collision terms, obtained by integrating out soft fluctuations around the mean fields, agrees with the QCD effective approaches [5,12]. The relationship between the transport theory with classical color and QCD can be found through the study of the quantum path integral within a saddle-point approximation [13].

In the second version of the QCD transport theory [7,14], the color charges are represented, in full accordance with $\mathrm{QCD}$, by a matrix structure of the distribution function. The Vlasov transport equation of quarks was derived [15] directly from $\mathrm{QCD}$, by analyzing the motion of quantum quarks in the classical chromodynamic field. The gluon transport equation was found [16,17], by splitting the gluon field into the mean field and the contribution representing the particle excitations. It was further observed [18] that the quark and gluon transport equations are formally identical when the first one is written in the fundamental representation and the second one in the adjoint representation. Then, the quark and gluon distribution functions are $N_{c} \times N_{c}$ and $\left(N_{c}^{2}-1\right) \times\left(N_{c}^{2}-1\right)$ matrices, respectively, for the $S U\left(N_{c}\right)$ gauge group. The early development of the quark-gluon kinetic theory was summarized at Ref. [19].

In quasiequilibrium, the matrix transport theory was proved [20] to be fully equivalent to the QCD hard-loop approach $[4,9,10]$. The kinetic approach, which can be treated as a local representation of the nonlocal hard-loop action, is particularly useful to study the collective excitations of the quark-gluon plasma, see [21] for a review. More recently, the quasiequilibrium kinetic equations have been derived beyond the collisionless limit [22] and the QCD effective theories [5] have again been correctly reproduced.

A natural question that arises is where the agreement between the kinetic theory, either with the classical color or in the matrix form, and the finite temperature diagrammatic approach breaks down. Surprisingly enough, the effective action of the static fields provided by the kinetic equations agrees with that obtained within perturbative QCD even at the $g^{3}$ order [23], where an operator responsible for $C$-odd processes appears for systems with finite baryon density. 
However, in a subsequent study [24] the kinetic theory with the classical color has been found to reproduce the $g^{4}$ contribution to the effective action only in the limit of highdimensional color representations. Thus, the limitations of the classical approach have been explicitly determined.

The aim of this paper is to clarify whether the difficulties faced by the classical color transport theory can be overcome when the matrix formulation is used. We explore how far the limits of the non-Abelian kinetic approach can be extended. For this purpose, we look for the solutions of the transport equations for quarks and gluons interacting with a chromodynamic mean field. The system is assumed to be translation invariant in one or more space-time directions. Thus, our considerations hold, in particular, for static and for homogeneous systems. We first present exact solutions when the vector gauge fields in the direction of the translation invariance commute with their covariant derivatives. Then, we use perturbation theory to solve the transport equation when the commutation condition is relaxed. Once the solutions are known, we derive the corresponding color current, and then the associated effective action. In the case of thermodynamic equilibrium, our results agree with those obtained by computing the one-loop effective action of QCD in the presence of a constant background field, which also corresponds to the effective potential of the dimensionally reduced theory [27]. However, for static and nonconstant background fields such that the commutation condition is not satisfied, we find additional nonlocal operators that correct the effective potential of [27], in what seems to be a discrepancy between the two approaches.

The paper is organized as follows. In Sec. II we review the transport theory approach we use in this paper. Exact solutions of the transport equations for translation invariant systems are discussed in Sec. III while in Sec. IV we solve the equations perturbatively. The effective actions for static and for homogeneous systems close to equilibrium are derived in Sec. V and we conclude our considerations in Sec. VI. The evaluation of some momentum integrals is left for Appendix A and we collect in Appendix B some formulas of the traces of $S U\left(N_{c}\right)$ generators in the adjoint representation. Finally, in Appendix C we briefly discuss the one-loop effective action $[2,25,26]$ which is compared to our results.

\section{TRANSPORT EQUATIONS}

In this section we briefly review the transport theory of quarks and gluons $[19,18]$. While we will restrict the discussion to QCD, with $N_{f}$ massless quarks and antiquarks carrying color in the fundamental representation and gluons in the adjoint, the results could easily be generalized to a different non-Abelian theory with different field content.

The distribution function of (anti)quarks $Q(p, x)$ $[\bar{Q}(p, x)]$ is a Hermitian $N_{c} \times N_{c}$ matrix in color space [for a $S U\left(N_{c}\right)$ color group]; $x$ denotes the space-time quark coordinate and $p$ its momentum, which is not constrained by the mass-shell condition. The spin of quarks and gluons is taken into account as an internal degree of freedom. The distribution function transforms under a local gauge transformation $M$ as

$$
Q(p, x) \rightarrow M(x) Q(p, x) M^{\dagger}(x) .
$$

Here and most cases below, the color indices are suppressed. The distribution function of hard gluons is a Hermitian $\left(N_{c}^{2}\right.$ $-1) \times\left(N_{c}^{2}-1\right)$ matrix, which transforms as

$$
G(p, x) \rightarrow \mathcal{M}(x) G(p, x) \mathcal{M}^{\dagger}(x),
$$

where

$$
\mathcal{M}_{a b}(x)=\operatorname{Tr}\left[\tau_{a} M(x) \tau_{b} M^{\dagger}(x)\right],
$$

with $\tau_{a}, a=1, \ldots, N_{c}^{2}-1$ being the $S U\left(N_{c}\right)$ group generators in the fundamental representation with $\operatorname{Tr}\left(\tau_{a} \tau_{b}\right)$ $=\frac{1}{2} \delta_{a b}$.

In a collisionless limit, the distribution functions of quarks and gluons satisfy the transport equations:

$$
\begin{aligned}
& p^{\mu} D_{\mu} Q(p, x)+\frac{g}{2} p^{\mu}\left\{F_{\mu \nu}(x), \frac{\partial Q(p, x)}{\partial p_{\nu}}\right\}=0, \\
& p^{\mu} D_{\mu} \bar{Q}(p, x)-\frac{g}{2} p^{\mu}\left\{F_{\mu \nu}(x), \frac{\partial \bar{Q}(p, x)}{\partial p_{\nu}}\right\}=0, \\
& p^{\mu} \mathcal{D}_{\mu} G(p, x)+\frac{g}{2} p^{\mu}\left\{\mathcal{F}_{\mu \nu}(x), \frac{\partial G(p, x)}{\partial p_{\nu}}\right\}=0,
\end{aligned}
$$

where $g$ is the QCD coupling constant, $\{\ldots, \ldots\}$ denotes the anticommutator; the covariant derivatives $D_{\mu}$ and $\mathcal{D}_{\mu}$ act as

$$
D_{\mu}=\partial_{\mu}-i g\left[A_{\mu}(x), \ldots\right], \quad \mathcal{D}_{\mu}=\partial_{\mu}-i g\left[\mathcal{A}_{\mu}(x), \ldots\right],
$$

$A_{\mu}$ and $\mathcal{A}_{\mu}$ being four-potentials in the fundamental and adjoint representations, respectively,

$$
A^{\mu}(x)=A_{a}^{\mu}(x) \tau_{a}, \quad \mathcal{A}_{a b}^{\mu}(x)=-i f_{a b c} A_{c}^{\mu}(x),
$$

and $f_{a b c}$ are the structure constants of the $S U\left(N_{c}\right)$ group. Since the generators of $S U\left(N_{c}\right)$ in the adjoint representation are given by $\left(T_{a}\right)_{b c}=-i f_{a b c}$, one can also write $\mathcal{A}^{\mu}$ $=A_{a}^{\mu} T^{a}$. The stress tensor in the fundamental representation is $F_{\mu \nu}=\partial_{\mu} A_{\nu}-\partial_{\nu} A_{\mu}-i g\left[A_{\mu}, A_{\nu}\right]$, while $\mathcal{F}_{\mu \nu}$ denotes the field strength tensor in the adjoint representation.

Sometimes it is convenient to project the matrix equations (2.3) into their colorless and colored components. For the quark distribution function we define

$$
Q(p, x)=\tilde{q}(p, x)+q^{a}(p, x) \tau^{a} .
$$

Then, we can deduce from Eq. (2.3a) a set of coupled equations for the different components of $Q$ defined by Eq. (2.4). More precisely, we find

$$
p^{\mu} \partial_{\mu} \tilde{q}(p, x)+\frac{g}{2 N_{c}} p^{\mu} F_{\mu \nu}^{a}(x) \frac{\partial q^{a}(p, x)}{\partial p_{\nu}}=0,
$$




$$
\begin{gathered}
p^{\mu} D_{\mu}^{a b} q^{b}(p, x)+\frac{g}{2} d^{a b c} p^{\mu} F_{\mu \nu}^{b}(x) \frac{\partial q^{c}(p, x)}{\partial p_{\nu}} \\
+g p^{\mu} F_{\mu \nu}^{a}(x) \frac{\partial \tilde{q}(p, x)}{\partial p_{\nu}}=0,
\end{gathered}
$$

where $d^{a b c}$ are the totally symmetric structure constants of $S U\left(N_{c}\right)$ and $D_{\mu}^{a c}=\partial_{\mu} \delta^{a c}+g f^{a b c} A_{\mu}^{b}$. Similar equations can be written for the antiquark and gluon distribution functions. Equations (2.5a), (2.5b) reflect in a very clear way that transport phenomena of colorless and colored fluctuations are coupled beyond the lowest order in the gauge coupling constant. Equations (2.5a), (2.5b) might be very useful when collisions are also taken into account. In this study, however, we find it more convenient to work with the matrix equations $(2.3 \mathrm{a})-(2.3 \mathrm{c})$

Once the solution of the transport equations is known, we can obtain the color current associated to the plasma constituents. The color current is expressed in the fundamental representation as

$$
\begin{aligned}
j^{\mu}(x)= & -\frac{g}{2} \int d P p^{\mu}\left[Q(p, x)-\bar{Q}(p, x)-\frac{1}{N_{c}} \operatorname{Tr}[Q(p, x)\right. \\
& \left.-\bar{Q}(p, x)]+2 i \tau_{a} f_{a b c} G_{b c}(p, x)\right]
\end{aligned}
$$

so that $j_{a}^{\mu}(x)=2 \operatorname{Tr}\left(\tau_{a} j^{\mu}(x)\right)$, and the momentum measure

$$
d P=\frac{d^{4} p}{(2 \pi)^{3}} 2 \Theta\left(p_{0}\right) \delta\left(p^{2}\right)
$$

takes into account the mass-shell condition $p_{0}=|\mathbf{p}|$. Throughout the paper, we neglect the quark masses, although those might easily be taken into account by modifying the mass-shell constraint in the momentum measure. A sum over helicities, two per particle, and over quark flavors $N_{f}$ is understood in Eq. (2.6), even though it is not explicitly written down.

In the transport theory framework one can consider two different physical situations: (1) the gauge fields entering into the transport equations $(2.3 \mathrm{a})-(2.3 \mathrm{c})$ are external, not due to the plasma constituents; (2) the gauge fields can be generated self-consistently by the quarks and gluons. In the latter case, one also has to solve the Yang-Mills equation

$$
D_{\mu} F^{\mu \nu}(x)=j^{\nu}(x),
$$

where the color current is given by Eq. (2.6).

The color current can be derived from an effective action added to the Yang-Mills one. By means of the relation

$$
j_{a}^{\mu}=-\frac{\delta S}{\delta A_{\mu}^{a}},
$$

where $S \equiv \int d^{4} x \mathcal{L}$, one can obtain the effective action, up to an integration constant, from the knowledge of the color cur- rent. In the remaining part of this article we will use this approach to obtain the effective action in different physical situations.

\section{EXACT SOLUTIONS}

Finding exact solutions of the transport equations (2.3a)(2.3c) is in general a difficult task. However, it is possible to find such solutions under some restrictive conditions. Here we consider a system where both the vector gauge field and the distribution functions are invariant under the space-time translation(s), i.e.

$$
\partial_{\alpha_{i}} A^{\mu}(x)=0, \quad \mu=0,1,2,3,
$$

and

$$
\partial_{\alpha_{i}} Q(p, x)=\partial_{\alpha_{i}} \bar{Q}(p, x)=\partial_{\alpha_{i}} G(p, x)=0
$$

for a fixed $\alpha_{i}$, where $\alpha_{i}$ can involve more than one Lorentz index. For example, if $\alpha_{i}=0$ the system is static while for $\alpha_{i}=1,2,3$ the gauge field and the distribution functions depend only on time. The condition Eq. (3.1) is a choice of gauge that, as we will show below, allows one to find solutions to the transport equations that respect the translation invariance of the system.

To solve Eqs. (2.3a)-(2.3c) for translation invariant systems along the direction $x^{\alpha_{i}}$, we will take into account two known facts. First, in an electromagnetic plasma, an exact solution of the corresponding transport equation is given by any function of the canonical momentum $p_{\alpha_{i}}-e A_{\alpha_{i}}(x)$, if $\partial_{\alpha_{i}} A_{\mu}=0$ [28]. Second, the non-Abelian transport equations of particles carrying a classical color charge $I^{a}$ for these translation invariant systems are also solved by any function of the canonical momentum $p_{\alpha_{i}}-g A_{\alpha_{i}}^{a}(x) I^{a}$, if $\partial_{\alpha_{i}} A_{\mu}^{a}=0$ [24]. Because the first case represents the Abelian limit of the transport equations $(2.3 \mathrm{a})-(2.3 \mathrm{c})$, while the second one corresponds to the limit of high-dimensional color representations, ${ }^{1}$ one expects that the solutions of Eqs. $(2.3 \mathrm{a})-(2.3 \mathrm{c})$ are of the form

$$
\begin{aligned}
Q(p, x)= & f\left(p_{\alpha_{i}}-g A_{\alpha_{i}}(x)\right) \\
= & \sum_{n=0}^{\infty} \frac{(-g)^{n}}{n !} A_{\alpha_{1}}(x) A_{\alpha_{2}}(x) \cdots A_{\alpha_{n}}(x) \\
& \times \frac{\partial^{n} f\left(p_{\alpha_{i}}\right)}{\partial p_{\alpha_{1}} \partial p_{\alpha_{2}} \ldots \partial p_{\alpha_{n}}},
\end{aligned}
$$

\footnotetext{
${ }^{1}$ While we are considering here the transport equations only for particles carrying color in the fundamental and adjoint representations, the equations are expected to have the same structure in any other non-Abelian representation.
} 


$$
\begin{aligned}
\bar{Q}(p, x)= & \bar{f}\left(p_{\alpha_{i}}+g A_{\alpha_{i}}(x)\right) \\
= & \sum_{n=0}^{\infty} \frac{g^{n}}{n !} A_{\alpha_{1}}(x) A_{\alpha_{2}}(x) \cdots A_{\alpha_{n}}(x) \\
& \times \frac{\partial^{n} \bar{f}\left(p_{\alpha_{i}}\right)}{\partial p_{\alpha_{1}} \partial p_{\alpha_{2}} \ldots \partial p_{\alpha_{n}}}, \\
G(p, x)= & f_{g}\left(p_{\alpha_{i}}-g \mathcal{A}_{\alpha_{i}}(x)\right) \\
= & \sum_{n=0}^{\infty} \frac{(-g)^{n}}{n !} \mathcal{A}_{\alpha_{1}}(x) \mathcal{A}_{\alpha_{2}}(x) \cdots \mathcal{A}_{\alpha_{n}}(x) \\
& \times \frac{\partial^{n} f_{g}\left(p_{\alpha_{i}}\right)}{\partial p_{\alpha_{1}} \partial p_{\alpha_{2}} \ldots \partial p_{\alpha_{n}}},
\end{aligned}
$$

where it is understood that a sum is taken over the repeated indices. The functions $f, \bar{f}$ and $f_{g}$ are, in principle, arbitrary but they can be fixed by additional considerations.

Let us note that the distribution functions given by Eqs. (3.3a)-(3.3c) transform covariantly, i.e. according to Eqs. (2.1) and (2.2), even though the potentials $A^{\mu}, \mathcal{A}^{\mu}$, in general, do not. Indeed,

$$
A^{\alpha_{i}}(x) \rightarrow M(x) A^{\alpha_{i}}(x) M^{\dagger}(x)-\frac{i}{g}\left(\partial^{\alpha_{i}} M(x)\right) M^{\dagger}(x) .
$$

However, the second term in the right-hand side (RHS) of Eq. (3.4), which transforms noncovariantly, is eliminated because of the condition (3.1).

Let us check under which conditions the ansatz, Eq. (3.3), solves the transport equations. We first note that Eq. (3.3a) is totally symmetric under the exchange of indices $\alpha_{1}, \ldots, \alpha_{n}$. Inserting Eq. (3.3a) into the transport equation (2.3a), one finds

$$
\begin{aligned}
p^{\mu} D_{\mu} Q & (p, x) \\
= & p^{\mu} \sum_{n=0}^{\infty} \frac{(-g)^{n}}{n !} \sum_{s=0}^{n-1} A_{\alpha_{1}} \cdots A_{\alpha_{s}}\left(D_{\mu} A_{\alpha_{s+1}}\right) \cdots A_{\alpha_{n}} \\
& \times \frac{\partial^{n} f\left(p_{\alpha_{i}}\right)}{\partial p_{\alpha_{1}} \partial p_{\alpha_{2}} \cdots \partial p_{\alpha_{n}}}, \\
\frac{g}{2} p^{\mu}\left\{F_{\mu \nu}(x), \frac{\partial Q(p, x)}{\partial p_{\nu}}\right\} & \\
= & -p^{\mu} \sum_{n=0}^{\infty} \frac{(-g)^{n+1}}{2 n !}\left\{D_{\mu} A_{\alpha_{i}}, A_{\alpha_{1}} \cdots A_{\alpha_{n}}\right\} \\
& \times \frac{\partial^{n+1} f\left(p_{\alpha_{i}}\right)}{\partial p_{\alpha_{i}} \partial p_{\alpha_{1}} \cdots \partial p_{\alpha_{n}}},
\end{aligned}
$$

where we have used the following property of a commutator:

$$
\left[X, Y^{n}\right]=\sum_{s=0}^{n-1} Y^{s}[X, Y] Y^{n-s-1}
$$

to derive Eq. (3.5). We have also taken into account that $F_{\mu \alpha_{i}}=D_{\mu} A_{\alpha_{i}}$ because of Eq. (3.1).

It is easy to observe that the terms in Eq. (3.5) and Eq. (3.6) that correspond to the same order of the derivative of $f$ cancel each other exactly if

$$
\left[D_{\mu} A_{\alpha_{i}}, A_{\alpha_{j}}\right]=0, \quad \mu=0,1,2,3,
$$

where $A_{\alpha_{j}}$ is also in the direction of the translation invariance. The same condition is obtained for the antiquark distribution function, while for gluons one finds that Eq. (3.3c) is an exact solution of Eq. (2.3c) if

$$
\left[\mathcal{D}_{\mu} \mathcal{A}_{\alpha_{i}}, \mathcal{A}_{\alpha_{j}}\right]=0, \quad \mu=0,1,2,3
$$

However, it is easy to prove that Eq. (3.8) is automatically satisfied if Eq. (3.7) holds.

For static systems $(\alpha=0)$, Eq. (3.7) reduces to the commutation relation between the color electric field and $A_{0}$. In a more general situation, the condition Eq. (3.7) simplifies the non-Abelian field dynamics in the direction of the translation invariance. Note that the commutation condition is trivially satisfied in the Abelian limit. If $T_{R}^{a}$ is a generator of a representation $R$ of $S U\left(N_{c}\right)$, then, after a normalization of these generators, one would get $\left[T_{R}^{a}, T_{R}^{b}\right] \rightarrow 0$ for highdimensional representations. Consequently, the commutation condition would also be satisfied. This explains how to reconcile the matrix results with those obtained with the nonAbelian transport equations for classical color.

Once the solution for the quark, antiquark and gluon distribution functions are known, one can compute the color current. Inserting Eqs. (3.3a)-(3.3c) into Eq. (2.6), we get

$$
\begin{aligned}
j_{a}^{\mu}(x)= & 2 \sum_{n=0}^{\infty} \frac{(-g)^{n+1}}{n !} A_{\alpha_{1}}^{c_{1}}(x) \cdots A_{\alpha_{n}}^{c_{n}}(x) \\
& \times \int d P p^{\mu}\left[N _ { f } \operatorname { T r } [ \tau _ { a } \tau _ { c _ { 1 } } \cdots \tau _ { c _ { n } } ] \left[\frac{\partial^{n} f\left(p_{\alpha_{i}}\right)}{\partial p_{\alpha_{1}} \cdots \partial p_{\alpha_{n}}}\right.\right. \\
& \left.+(-1)^{n+1} \frac{\partial^{n} \bar{f}\left(p_{\alpha_{i}}\right)}{\partial p_{\alpha_{1}} \cdots \partial p_{\alpha_{n}}}\right] \\
& \left.+\operatorname{Tr}\left[T_{a} T_{c_{1}} \cdots T_{c_{n}}\right] \frac{\partial^{n} f_{g}\left(p_{\alpha_{i}}\right)}{\partial p_{\alpha_{1}} \cdots \partial p_{\alpha_{n}}}\right]
\end{aligned}
$$

The factor 2 in the above equation arises from the two helicities associated with every particle species. When the functions $f, \bar{f}$ and $f_{g}$ are determined, one can evaluate the momentum integral of Eq. (3.9), and then, after solving Eq. (2.9), one obtains the associated effective action. 


\section{PERTURBATIVE SOLUTIONS}

\section{A. General considerations}

In Sec. III we have found exact solutions of the collisionless transport equations $(2.3 \mathrm{a})-(2.3 \mathrm{c})$ for translation invariant systems that obey the extra condition Eq. (3.7). In this section we treat the transport equations perturbatively and find solutions that are not constrained by the condition Eq. (3.7).

We assume here that

$$
g \ll 1,
$$

i.e. we deal with the weak coupling regime of the theory, and consider an expansion of the distribution function of the form

$$
Q=Q^{(0)}+Q^{(1)}+Q^{(2)}+\ldots
$$

where the 0 th term is a known function

$$
Q^{(0)}(p, x)=f\left(p_{\alpha_{i}}\right)
$$

The higher-order terms are determined by the equation

$$
p^{\mu} D_{\mu} Q^{(n)}(p, x)+\frac{g}{2} p^{\mu}\left\{F_{\mu \nu}(x), \frac{\partial Q^{(n-1)}(p, x)}{\partial p_{\nu}}\right\}=0 .
$$

Of course, the same treatments should be followed to study the antiquark and gluon distribution functions, but those are nearly identical.

The iterative procedure based on Eq. (4.4) does not correspond to a strict expansion in powers of $g$. Such an expansion would force us to split all covariant derivatives into a derivative and a commutator part, resulting in the breaking of the gauge covariance of every term in the perturbative series. We maintain the gauge covariance of every term in Eq. (4.2) at the expense of reorganizing the perturbative expansion.

It should be noticed that for $f$ being the equilibrium distribution function, the first term $(n=1)$ of the above perturbation series reproduces the hard thermal loops of QCD $[4,20]$. Let us also stress that while we are applying here a perturbative method to the transport equations in their matrix form, fully equivalent results can be obtained using the projected equations (2.5a), (2.5b). Such an approach has been carried out by Bödeker and Laine to order $g^{2}$ for static quasiequilibrium systems [23]. We extend that analysis by pushing the perturbative procedure to higher orders in $g$. We do not attempt to solve the transport equations in full generality, as the solutions at every order then turn out to be highly nonlocal. We reduce our study to translation invariant systems when the solutions are much simplified.

\section{B. From $g$ to $g^{4}$ order}

In this subsection, we consider a translation invariant system that obeys Eq. (3.1) for a fixed $\alpha_{i}$. We also assume that the unperturbed distribution function depends only on $p_{\alpha_{i}}$ as in Eq. (4.3). The first-order correction to $Q^{(0)}$ is obtained by solving the equation

$$
p^{\mu} D_{\mu} Q^{(1)}=-g p^{\mu} F_{\mu \alpha_{1}} \frac{\partial f\left(p_{\alpha_{i}}\right)}{\partial p_{\alpha_{1}}}=-g p^{\mu} D_{\mu} A_{\alpha_{1}} \frac{\partial f\left(p_{\alpha_{i}}\right)}{\partial p_{\alpha_{1}}} .
$$

The solution reads (up to a function $h$, such that $p^{\mu} D_{\mu} h$ $=0$, that we will neglect throughout)

$$
Q^{(1)}(p, x)=-g A_{\alpha_{1}}(x) \frac{\partial f\left(p_{\alpha_{i}}\right)}{\partial p_{\alpha_{1}}}
$$

and it coincides with the first term of the ansatz (3.3a).

The transport equation at second order is

$$
p^{\mu} D_{\mu} Q^{(2)}=\frac{g^{2}}{2}\left\{p^{\mu} D_{\mu} A_{\alpha_{1}}, A_{\alpha_{2}}\right\} \frac{\partial^{2} f\left(p_{\alpha_{i}}\right)}{\partial p_{\alpha_{1}} \partial p_{\alpha_{2}}},
$$

but it can be rewritten as

$$
p^{\mu} D_{\mu} Q^{(2)}=\frac{g^{2}}{2} p^{\mu} D_{\mu}\left(A_{\alpha_{1}} A_{\alpha_{2}}\right) \frac{\partial^{2} f\left(p_{\alpha_{i}}\right)}{\partial p_{\alpha_{1}} \partial p_{\alpha_{2}}} .
$$

Thus, the second-order solution is

$$
Q^{(2)}(p, x)=\frac{g^{2}}{2} A_{\alpha_{1}}(x) A_{\alpha_{2}}(x) \frac{\partial^{2} f\left(p_{\alpha_{i}}\right)}{\partial p_{\alpha_{1}} \partial p_{\alpha_{2}}},
$$

which again coincides with the respective term of the ansatz (3.3a).

The third-order equation is

$$
p^{\mu} D_{\mu} Q^{(3)}=-\frac{g^{3}}{4}\left\{p^{\mu} D_{\mu} A_{\alpha_{1}}, A_{\alpha_{2}} A_{\alpha_{3}}\right\} \frac{\partial^{3} f\left(p_{\alpha_{i}}\right)}{\partial p_{\alpha_{1}} \partial p_{\alpha_{2}} \partial p_{\alpha_{3}}}
$$

and its RHS is not proportional to a covariant derivative of a third power of $A_{\alpha_{i}}$. However, the anticommutator of the RHS of the equation can be rewritten as follows:

$$
\begin{aligned}
p^{\mu} D_{\mu} Q^{(3)}= & -\frac{g^{3}}{3 !}\left(p^{\mu} D_{\mu}\left(A_{\alpha_{1}} A_{\alpha_{2}} A_{\alpha_{3}}\right)\right. \\
& \left.+\frac{1}{2}\left[\left[p^{\mu} D_{\mu} A_{\alpha_{1}}, A_{\alpha_{2}}\right], A_{\alpha_{3}}\right]\right) \frac{\partial^{3} f\left(p_{\alpha_{i}}\right)}{\partial p_{\alpha_{1}} \partial p_{\alpha_{2}} \partial p_{\alpha_{3}}} .
\end{aligned}
$$

The term with the commutator cannot be expressed as a total covariant derivative. Thus, the solution of the above equation 
contains two pieces: a term that is local in the gauge fields and a nonlocal part. Namely,

$$
\begin{aligned}
Q^{(3)}(x, p)= & -\frac{g^{3}}{3 !} A_{\alpha_{1}}(x) A_{\alpha_{2}}(x) A_{\alpha_{3}}(x) \frac{\partial^{3} f\left(p_{\alpha_{i}}\right)}{\partial p_{\alpha_{1}} \partial p_{\alpha_{2}} \partial p_{\alpha_{3}}} \\
& +Q_{\mathrm{nl}}^{(3)}(x, p)
\end{aligned}
$$

with the non-local term obeying the equation

$$
p^{\mu} D_{\mu} Q_{\mathrm{nl}}^{(3)}=-\frac{g^{3}}{12}\left[\left[p^{\mu} D_{\mu} A_{\alpha_{1}}, A_{\alpha_{2}}\right], A_{\alpha_{3}}\right] \frac{\partial^{3} f\left(p_{\alpha_{i}}\right)}{\partial p_{\alpha_{1}} \partial p_{\alpha_{2}} \partial p_{\alpha_{3}}} .
$$

One observes that when the condition Eq. (3.7) is satisfied, $Q_{\mathrm{nl}}^{(3)}=0$ and we recover the solution of order $g^{3}$ of Eq. (3.3a). But this term is nonzero under more general circumstances. We also notice that if

$$
\left[\partial_{\mu} A_{\alpha_{i}}, A_{\alpha_{j}}\right]=0
$$

the nonlocal piece is proportional to $g^{4}$.

Let us solve Eq. (4.13). Using the $S U\left(N_{c}\right)$ algebra, we rewrite the commutator as

$$
\begin{aligned}
p^{\mu} D_{\mu} Q_{\mathrm{nl}}^{(3)}= & -\frac{g^{3}}{12} f^{a c d} f^{d e b} \tau^{a}\left(p^{\mu} D_{\mu} A_{\alpha_{1}}\right)^{e} A_{\alpha_{2}}^{c} A_{\alpha_{3}}^{b} \\
& \times \frac{\partial^{3} f\left(p_{\alpha_{i}}\right)}{\partial p_{\alpha_{1}} \partial p_{\alpha_{2}} \partial p_{\alpha_{3}}} \\
\equiv & R^{a} \tau^{a} .
\end{aligned}
$$

Consequently, $Q_{\mathrm{nl}}^{(3)}=Q_{\mathrm{nl}}^{(3) a} \tau^{a}$ with

$$
p^{\mu} D_{\mu}^{a c} Q_{\mathrm{nl}}^{(3) c}=R^{a} .
$$

The solution can be expressed as

$$
Q_{\mathrm{nl}}^{(3) a}(p, x)=\int d^{4} y\left\langle x\left|\frac{1}{p \cdot D}\right| y\right\rangle_{a b} R^{b}(y),
$$

where $1 / p \cdot D$ is the retarded Green function associated to the differential equation (4.16). The explicit form of $1 / p \cdot D$ can be found, for example, in Sec. II of [29].

The fourth-order equation reads

$$
\begin{aligned}
p^{\mu} D_{\mu} Q^{(4)}= & \frac{g^{4}}{12}\left\{p^{\mu} D_{\mu} A_{\alpha_{1}}, A_{\alpha_{2}} A_{\alpha_{3}} A_{\alpha_{4}}\right\} \frac{\partial^{4} f\left(p_{\alpha_{i}}\right)}{\partial p_{\alpha_{1}} \partial p_{\alpha_{2}} \partial p_{\alpha_{3}} \partial p_{\alpha_{4}}} \\
& +\frac{g^{4}}{24}\left\{F_{\mu \nu}, \frac{\partial Q_{\mathrm{nl}}^{(3)}}{\partial p_{\nu}}\right\} .
\end{aligned}
$$

The first anticommutator in the RHS of the equation can also be rewritten as a total covariant derivative plus an additional commutator. Thus,

$$
\begin{aligned}
p^{\mu} D_{\mu} Q^{(4)}= & \frac{g^{4}}{4 !}\left(p^{\mu} D_{\mu}\left(A_{\alpha_{1}} A_{\alpha_{2}} A_{\alpha_{3}} A_{\alpha_{4}}\right)\right. \\
& \left.+\left[\left[p^{\mu} D_{\mu} A_{\alpha_{1}}, A_{\alpha_{2}}\right], A_{\alpha_{3}} A_{\alpha_{4}}\right]\right) \\
& \times \frac{\partial^{4} f\left(p_{\alpha_{i}}\right)}{\partial p_{\alpha_{1}} \partial p_{\alpha_{2}} \partial p_{\alpha_{3}} \partial p_{\alpha_{4}}}+\frac{g^{4}}{4 !}\left\{F_{\mu \nu}, \frac{\partial Q_{\mathrm{nl}}^{(3)}}{\partial p_{\nu}}\right\} .
\end{aligned}
$$

We see that the solution is of the form

$$
\begin{aligned}
Q^{(4)}(x, p)= & \frac{g^{4}}{4 !} A_{\alpha_{1}}(x) A_{\alpha_{2}}(x) A_{\alpha_{3}}(x) A_{\alpha_{4}}(x) \\
& \times \frac{\partial^{4} f\left(p_{\alpha_{i}}\right)}{\partial p_{\alpha_{1}} \partial p_{\alpha_{2}} \partial p_{\alpha_{3}} \partial p_{\alpha_{4}}}+Q_{\mathrm{nl}}^{(4)}(x, p),
\end{aligned}
$$

where $Q_{\mathrm{nl}}^{(4)}$ represents the nonlocal contribution that vanishes if the condition Eq. (3.7) is satisfied. We note that the local piece coincides with the term $g^{4}$ of the ansatz (3.3a).

In principle, we could solve the equation for $Q_{\mathrm{nl}}^{(4)}$ and for the following terms of the perturbative expansion, finding that every term contains both local and nonlocal pieces in the gauge fields. However, we stop our analysis here as the structure of the nonlocal terms becomes more and more complex.

Adding the contributions of quarks, antiquarks and gluons, we can obtain the color current. Up to the term $n=3$, the color current still has a relatively simple form and is given by

$$
\begin{aligned}
j_{a}^{\mu}(x)= & 2 \sum_{n=0}^{3} \frac{(-g)^{n+1}}{n !} A_{\alpha_{1}}^{c_{1}}(x) \cdots A_{\alpha_{n}}^{c_{n}}(x) \\
& \times \int d P p^{\mu}\left[N _ { f } \operatorname { T r } [ \tau _ { a } \tau _ { c _ { 1 } } \cdots \tau _ { c _ { n } } ] \left[\frac{\partial^{n} f\left(p_{\alpha_{i}}\right)}{\partial p_{\alpha_{1}} \cdots \partial p_{\alpha_{n}}}\right.\right. \\
& \left.+(-1)^{n+1} \frac{\partial^{n} \bar{f}\left(p_{\alpha_{i}}\right)}{\partial p_{\alpha_{1}} \cdots \partial p_{\alpha_{n}}}\right] \\
& \left.+\operatorname{Tr}\left[T_{a} T_{c_{1}} \cdots T_{c_{n}}\right] \frac{\partial^{n} f_{g}\left(p_{\alpha_{i}}\right)}{\partial p_{\alpha_{1}} \cdots \partial p_{\alpha_{n}}}\right]+j_{a, \mathrm{nl}}^{\mu}(x),
\end{aligned}
$$

where the nonlocal term reads 


$$
\begin{aligned}
j_{a, \mathrm{nl}}^{\mu}(x)= & \frac{g^{4}}{6} \int d P \int d^{4} y\left\langle x\left|\frac{p^{\mu}}{p \cdot D}\right| y\right\rangle_{a b} \\
& \times\left[2 N_{f} \operatorname{Tr}\left(\tau^{b}\left[\left[p \cdot D A_{\alpha_{1}}, A_{\alpha_{2}}\right], A_{\alpha_{3}}\right]\right)\right. \\
& \times\left[\frac{\partial^{3} f\left(p_{\alpha_{i}}\right)}{\partial p_{\alpha_{1}} \partial p_{\alpha_{2}} \partial p_{\alpha_{3}}}+\frac{\partial^{3} \bar{f}\left(p_{\alpha_{i}}\right)}{\partial p_{\alpha_{1}} \partial p_{\alpha_{2}} \partial p_{\alpha_{3}}}\right] \\
& +\frac{1}{N_{c}} \operatorname{Tr}\left(T^{b}\left[\left[p \cdot \mathcal{D} \mathcal{A}_{\alpha_{1}}, \mathcal{A}_{\alpha_{2}}\right], \mathcal{A}_{\alpha_{3}}\right]\right) \\
& \left.\times \frac{\partial^{3} f_{g}\left(p_{\alpha_{i}}\right)}{\partial p_{\alpha_{1}} \partial p_{\alpha_{2}} \partial p_{\alpha_{3}}}\right] .
\end{aligned}
$$

In the following section we will get the effective action for static systems close to equilibrium. In that case, it is easy to see that for $n \geqslant 4$ the local pieces do not contribute to the current or effective action, as either the momentum integrals (Appendix A) or the traces of generators in the adjoint representation (Appendix B) vanish. However, the same does not hold true for the nonlocal terms.

\section{EFFECTIVE ACTION OF QUASIEQUILIBRIUM SYSTEMS}

In this section we find the effective action for systems close to thermal equilibrium. Then, the functions $f, \bar{f}$ and $f_{g}$ are of the Fermi-Dirac or Bose-Einstein form

$$
\begin{gathered}
f_{\mathrm{FD}}(E)=\frac{1}{e^{\beta(E-\mu)}+1}, \quad \bar{f}_{\mathrm{FD}}(E)=\frac{1}{e^{\beta(E+\mu)}+1}, \\
f_{\mathrm{BE}}(E)=\frac{1}{e^{\beta E}-1},
\end{gathered}
$$

where $\beta \equiv 1 / T$, and $T$ is the temperature, and $\mu$ is the quark chemical potential.

\section{A. Static systems}

We first consider static systems satisfying the condition

$$
\left[D_{\mu} A_{0}, A_{0}\right]=0, \quad \mu=0,1,2,3 .
$$

The color current is given by Eq. (3.9) with $\alpha_{i}=0$ while the distribution functions are those in Eq. (5.1).

After performing the momentum integral, one observes that $j_{a}^{i}=0$ since

$$
\int \frac{d \Omega \mathbf{v}}{4 \pi} v^{i}=0
$$

where $v^{i}=p^{i} /|\mathbf{p}|$ is the particle velocity, and the integral is performed over angular directions of $\mathbf{v}$. Thus, only $j_{a}^{0}$, i.e. the color current density, is nonvanishing. In the case of static systems, one easily finds the Lagrangian density by integrating Eq. (2.9). The fermionic contribution arises from

$$
\begin{aligned}
\mathcal{L}_{f}= & -\frac{N_{f}}{\pi^{2}} \sum_{n=0}^{\infty} \frac{(-g)^{n+1}}{(n+1) !} \int_{\Lambda}^{\infty} d E E^{2}\left[\frac{d^{n} f_{\mathrm{FD}}(E)}{d E^{n}}\right. \\
& \left.+(-1)^{n+1} \frac{d^{n} \bar{f}_{\mathrm{FD}}(E)}{d E^{n}}\right] \operatorname{Tr}\left[A_{0}^{n+1}(\mathbf{x})\right],
\end{aligned}
$$

while that of gluons is

$$
\mathcal{L}_{g}=-\frac{1}{\pi^{2}} \sum_{n=0}^{\infty} \frac{(-g)^{n+1}}{(n+1) !} \int_{\Lambda}^{\infty} d E E^{2} \frac{d^{n} f_{\mathrm{BE}}(E)}{d E^{n}} \operatorname{Tr}\left[\mathcal{A}_{0}^{n+1}(\mathbf{x})\right]
$$

In the above expressions we have made use of the mass-shell condition, which gives $E \equiv p_{0}=|\mathbf{p}|$, and we have introduced an infrared cutoff, $T \gg \Lambda \gg g T$, which excludes the contribution of the soft modes to the integrals, as only the hard modes behave as quasiparticles.

We note that the above Lagrangians can be written as

$$
\begin{aligned}
\mathcal{L}_{f}= & \frac{T N_{f}}{\pi^{2}} \operatorname{Tr} \int_{\Lambda}^{\infty} d E E^{2}\left[\ln \left(1+e^{-\beta\left(E-\mu-g A^{0}\right)}\right)\right. \\
& \left.+\ln \left(1+e^{-\beta\left(E+\mu+g A^{0}\right)}\right)\right], \\
\mathcal{L}_{g}= & \frac{T}{\pi^{2}} \operatorname{Tr} \int_{\Lambda}^{\infty} d E E^{2} \ln \left(1-e^{-\beta\left(E-g \mathcal{A}^{0}\right)}\right),
\end{aligned}
$$

because

$$
\frac{d}{d x} \ln \left(1 \pm e^{-x}\right)= \pm \frac{1}{e^{x} \pm 1}
$$

The Lagrangians (5.4), (5.5) differ from those given in Eqs. (5.6), (5.7) by field independent terms corresponding to the free-quark and free-gluon contributions to the system's pressure.

While it is not obvious from Eq. (5.5), Eq. (5.7) shows that the gluon action is not well defined without the infrared cutoff because for energies smaller than the eigenvalues of $g A_{0}$, which are typically of order $g T$, the logarithm in Eq. (5.7) becomes a multivalued complex function. Of course, there is an analogous problem with the Lagrangian Eq. (5.5). The difficulty one encounters is not surprising - the kinetic description breaks down for sufficiently soft modes. Then, the equilibrium distribution function of gluons of the form (3.3c) becomes negative, losing its probabilistic interpretation.

We leave the evaluation of the energy integrals from Eqs. (5.4) and (5.5) for Appendix A, where we first compute the integrals with the cutoff. However, for all contributions the cutoff dependent terms appear to be regular and subdominant. Therefore, we take the limit $\Lambda \rightarrow 0$ and thus drop all the cutoff dependent terms. ${ }^{2}$ As a result, we find the field dependent fermionic contribution to the system's Lagrangian as

\footnotetext{
${ }^{2} \mathrm{~A}$ matching procedure with the soft classical field theory allows one to eliminate the cutoff dependent terms but we will not pursue that procedure here.
} 


$$
\begin{aligned}
\frac{\mathcal{L}_{f}}{N_{f}}= & g \frac{\mu}{3}\left(T^{2}+\frac{\mu^{2}}{\pi^{2}}\right) \operatorname{Tr} A_{0}+\frac{g^{2}}{2}\left(\frac{T^{2}}{3}+\frac{\mu^{2}}{\pi^{2}}\right) \operatorname{Tr} A_{0}^{2} \\
& +\mu \frac{g^{3}}{3 \pi^{2}} \operatorname{Tr} A_{0}^{3}+\frac{g^{4}}{12 \pi^{2}} \operatorname{Tr} A_{0}^{4} .
\end{aligned}
$$

We have kept here a linear term in the gauge potential that only survives in the Abelian limit where $\operatorname{Tr} A_{0}=A_{0}$. Taking into account that, as shown in Appendix B, the symmetric traces of an odd number of adjoint generators vanish, we find the following gluon contribution

$$
\mathcal{L}_{g}=\frac{g^{2} T^{2}}{6} \operatorname{Tr} \mathcal{A}_{0}^{2}-\frac{g^{4}}{24 \pi^{2}} \operatorname{Tr} \mathcal{A}_{0}^{4} .
$$

It is also shown in Appendix B that the traces of the fields in the adjoint representation can be expressed through the fundamental representation traces.

As already stressed, the integral (5.7) is ill defined without the infrared cutoff. However, the integral with $\Lambda=0$ is regular after a Wick rotation to Euclidean space-time. For constant background fields $A_{0}$, and after the Wick rotation $A_{0} \rightarrow i A_{0}^{E}$ we observe that Eqs. (5.6) and (5.7) with $\Lambda=0$ fully agree with the complete one-loop contribution to the effective potential for the phase of the Polyakov line $[2,25,26]$. It should be mentioned that a constant background field $A_{0}$ can always be chosen in diagonal form [2], and thus satisfies the condition Eq. (5.2). The Wick rotated integral (5.7) is discussed in Appendix C. We note here that except for the terms analogous to those present in Eq. (5.9) it provides non-analytic terms which are cubic in the eigenvalues of $A_{0}^{E}$. We also observe that Eqs. (5.8) and (5.9) after the Wick rotation agree with the dimensionally reduced effective action [27].

Now, let us briefly consider a static system that does not satisfy Eq. (5.2). Using the solutions of Sec. IV B, we find that up to the order $g^{4}$ Eqs. (5.8) and (5.9) still hold, but at order $g^{4}$ and beyond there are corrections due to the nonlocal terms. The first nonlocal contribution appears at order $g^{4}$ from the nonlocal current Eq. (4.22) with $\alpha_{i}=0$. To get the corresponding term in the effective action, one should still solve Eq. (2.9). We have not found the solution, but we also see no reason why the term should vanish. Thus, we conclude that the presence of the nonlocal contribution signals a discrepancy with the dimensionally reduced effective action [27] at order $g^{4}$, whenever the condition Eq. (5.2) is not satisfied.

\section{B. Homogeneous systems}

We consider here time-dependent homogeneous systems that obey the condition Eq. (3.7) with $\alpha_{i}=1,2,3$. For a system close to equilibrium, where the rotational symmetry is not broken, the solution of the transport equations can only depend on the modulus of the canonical three-momentum $|\mathbf{p}-g \mathbf{A}(t)|$. The color current in this situation is given by Eq. (3.9) with $f\left(p_{\alpha_{i}}\right), \bar{f}\left(p_{\alpha_{i}}\right)$ and $f_{g}\left(p_{\alpha_{i}}\right)$ replaced by $f_{\mathrm{FD}}(|\mathbf{p}|), \bar{f}_{\mathrm{FD}}(|\mathbf{p}|)$, and $f_{\mathrm{BE}}(|\mathbf{p}|)$, respectively.

The color current is then given by Eq. (3.9), but now $\alpha_{i}$ $=1,2,3$. To get the expression for the color current, we first need to perform the momentum integral of Eq. (3.9). The partial derivatives appearing in Eq. (3.9) can be explicitly evaluated. Using the chain rule, they can be expressed as partial derivatives of $E=|\mathbf{p}|$. Therefore,

$$
\begin{gathered}
\frac{\partial f}{\partial p_{i}}=\frac{\partial E}{\partial p_{i}} \frac{d f}{d E}, \frac{\partial^{2} f}{\partial p_{i} \partial p_{j}}=\frac{\partial^{2} E}{\partial p_{i} \partial p_{j}} \frac{d f}{d E}+\frac{\partial E}{\partial p_{i}} \frac{\partial E}{\partial p_{j}} \frac{d^{2} f}{d E^{2}} \\
\frac{\partial^{3} f}{\partial p_{i} \partial p_{j} \partial p_{k}}=\frac{\partial^{3} E}{\partial p_{i} \partial p_{j} \partial p_{k}} \frac{d f}{d E}+\left(\frac{\partial E}{\partial p_{i}} \frac{\partial^{2} E}{\partial p_{j} \partial p_{k}}+\frac{\partial E}{\partial p_{j}} \frac{\partial^{2} E}{\partial p_{i} \partial p_{k}}+\frac{\partial E}{\partial p_{k}} \frac{\partial^{2} E}{\partial p_{i} \partial p_{j}}\right) \frac{d^{2} f}{d E^{2}}+\frac{\partial E}{\partial p_{i}} \frac{\partial E}{\partial p_{j}} \frac{\partial E}{\partial p_{k}} \frac{d^{3} f}{d E^{3}}, \quad \text { etc. }
\end{gathered}
$$

where $i, j, k=1,2,3$. The partial derivatives of $E$ can be written as functions of the particle velocities. Let us define

$$
L_{(n)}^{i_{1} \cdots i_{n}} \equiv \frac{\partial^{n} E}{\partial p_{i_{1}} \cdots \partial p_{i_{n}}} .
$$

An explicit evaluation of the first terms gives

$$
\begin{gathered}
L_{(1)}^{i}=v^{i}, \quad L_{(2)}^{i j}=\frac{1}{E}\left(\delta^{i j}-v^{i} v^{j}\right), \\
L_{(3)}^{i j k}=-\frac{1}{E}\left(L_{(2)}^{i j} v^{k}+L_{(2)}^{i k} v^{j}+L_{(2)}^{j k} v^{i}\right), \quad \text { etc. }
\end{gathered}
$$

The angular integral of every term in the series of Eq. (3.9) can be easily calculated. Since the integrals of an even number of $v^{i}$ vanish we only need

$$
\int \frac{d \Omega_{\mathbf{v}}}{4 \pi} v^{i} v^{j}=\frac{1}{3} \delta^{i j}
$$

$$
\int \frac{d \Omega_{\mathbf{v}}}{4 \pi} v^{i} v^{j} v^{k} v^{l}=\frac{1}{15}\left(\delta^{i j} \delta^{k l}+\delta^{i k} \delta^{j l}+\delta^{i l} \delta^{j k}\right)
$$

The integrals over $E$ are the same as those used in the previous subsection (see Appendix A). 
The evaluation of the momentum integrals gives, as expected, $j_{a}^{0}=0$. The space components of the color current are, of course, nonzero with the quark and gluon contributions given, respectively, as

$$
\begin{aligned}
j_{a}^{j}= & \frac{g^{2}}{3}\left(\frac{T^{2}}{3}+\frac{\mu^{2}}{\pi^{2}}\right) \operatorname{Tr}\left(\tau_{a} A^{j}\right)+\frac{g^{4}}{45 \pi^{2}}\left(\operatorname{Tr}\left(\tau_{a} A^{j} A^{i} A^{i}\right)\right. \\
& \left.+\operatorname{Tr}\left(\tau_{a} A^{i} A^{j} A^{i}\right)+\operatorname{Tr}\left(\tau_{a} A^{i} A^{i} A^{j}\right)\right), \\
j_{a}^{j}= & \frac{g^{2} T^{2}}{9} \operatorname{Tr}\left(T_{a} \mathcal{A}\right)+\frac{g^{4}}{90 \pi^{2}}\left(\operatorname{Tr}\left(T_{a} \mathcal{A}^{j} \mathcal{A}^{i} \mathcal{A}^{i}\right)\right. \\
& \left.+\operatorname{Tr}\left(T_{a} \mathcal{A}^{i} \mathcal{A}^{j} \mathcal{A}^{i}\right)+\operatorname{Tr}\left(T_{a} \mathcal{A}^{i} \mathcal{A}^{i} \mathcal{A}^{j}\right)\right) .
\end{aligned}
$$

These currents arise from the following Lagrangian densities:

$$
\begin{aligned}
\frac{\mathcal{L}_{f}}{N_{f}}= & -\frac{g^{2}}{6}\left(\frac{T^{2}}{3}+\frac{\mu^{2}}{\pi^{2}}\right) \operatorname{Tr}\left(A_{j} A_{j}\right)-\frac{g^{4}}{90 \pi^{2}}\left(\operatorname{Tr}\left(A_{j} A_{j} A_{i} A_{i}\right)\right. \\
& \left.+\frac{1}{2} \operatorname{Tr}\left(A_{j} A_{i} A_{j} A_{i}\right)\right)
\end{aligned}
$$

and

$$
\begin{aligned}
\mathcal{L}_{g}= & -\frac{g^{2} T^{2}}{18} \operatorname{Tr}\left(\mathcal{A}_{j} \mathcal{A}_{j}\right)-\frac{g^{4}}{180 \pi^{2}}\left(\operatorname{Tr}\left(\mathcal{A}_{j} \mathcal{A}_{j} \mathcal{A}_{i} \mathcal{A}_{i}\right)\right. \\
& \left.+\frac{1}{2} \operatorname{Tr}\left(\mathcal{A}_{i} \mathcal{A}_{j} \mathcal{A}_{i} \mathcal{A}_{j}\right)\right) .
\end{aligned}
$$

We should point out that the terms proportional to $g^{2}$ in Eq. (5.15) and Eq. (5.16) agree with the hard thermal loop Lagrangians in the homogeneous limit [4]. If the condition Eq. (3.7) is not satisfied, the color current, and thus the associated effective Lagrangians, are corrected at order $g^{4}$ and beyond by the addition of the nonlocal terms, exactly as happened for the static systems. However, we will not write down these explicit terms here.

\section{CONCLUSIONS}

Our results show the efficiency of transport theory in describing the quark-gluon plasma at soft scales. We have shown how the solutions of the collisionless transport equations for the static systems close to equilibrium reproduce the one-loop effective potential for the phase of the Polyakov line. Up to now, the results of the transport theory and quantum field theoretical computations have been known to agree only for the lower-dimensional operators, but our computations indicate that the agreement extends to the full one-loop effective action. We find a complete match of the transport results with those of the one-loop effective potential in the presence of a constant background field. For nonconstant static background fields, transport theory predicts the appearance of nonlocal operators in the effective action, starting at order $g^{4}$ and beyond. This result then suggests a discrepancy with the dimensionally reduced effective theories [27], where these nonlocal operators are not present.

We have limited our analysis to translation invariant systems, when the solutions of the transport equations are local in the lower orders of the perturbative expansion. It would be desirable to solve the equations in full generality. However, the solutions are then complex nonlocal functions of the gauge fields, and their structure beyond order $g^{2}$ is not particularly enlightening. It is presumably more promising to explore the combined set of equations (2.3a)-(2.3c) and (2.8) numerically, thus allowing for a nonperturbative study of dynamical phenomena at soft scales, even beyond the hard thermal loop approximation.

\section{ACKNOWLEDGMENTS}

We wish to thank M. Laine for his critical reading of the manuscript and fruitful discussions. We are also indebted to J.L.F. Barbón, M. García-Perez, K. Korthals Altes and O. Philipsen for useful conversations. C.M. was partially supported by the EU through Contract No. HPMF-CT-199900391. Our special thanks go to the ITP at Santa Barbara where the project was initiated during the Workshop "QCD and Gauge Theory Dynamics in the RHIC Era." We are grateful to the NSF for support under Grant No. PHY9907949.

\section{APPENDIX A: MOMENTUM INTEGRALS}

\section{Bosons}

The bosonic integrals to be evaluated are of the form

$$
I_{n}^{b}(\Delta)=T^{3-n} \int_{\Delta}^{\infty} d x x^{2} \frac{d^{n} f_{\mathrm{BE}}(x)}{d x^{n}},
$$

where $\Delta \equiv \Lambda / T$. Expanding the Bose-Einstein distribution as

$$
f_{\mathrm{BE}}(x)=\frac{1}{e^{x}-1}=\sum_{m=1}^{\infty} e^{-m x}
$$

and interchanging the order of summation and integration, the integral that has to be performed reduces to

$$
\int_{\Delta}^{\infty} d x x^{2} e^{-m x}=e^{-m \Delta}\left(\frac{2}{m^{3}}+\frac{2 \Delta}{m^{2}}+\frac{\Delta^{2}}{m}\right) .
$$

Therefore,

$$
\begin{aligned}
I_{n}^{b}(\Delta)= & 2(-1)^{n} T^{3-n}\left(\operatorname{Li}_{3-n}\left(e^{-\Delta}\right)+\Delta \mathrm{Li}_{2-n}\left(e^{-\Delta}\right)\right. \\
& \left.+\frac{\Delta^{2}}{2} \operatorname{Li}_{1-n}\left(e^{-\Delta}\right)\right)
\end{aligned}
$$

where the solution is expressed in terms of the Euler polylogarithm function

$$
\operatorname{Li}_{s}(z) \stackrel{\operatorname{def}}{=} \sum_{m=1}^{\infty} \frac{z^{m}}{m^{s}} .
$$


The function $\operatorname{Li}_{N}\left(e^{-\Delta}\right)$ can be expanded in powers of $\Delta$ as [30,31]

$$
\operatorname{Li}_{N}\left(e^{-\Delta}\right)=\sum_{k=0}^{\infty}(-1)^{k} \frac{\Delta^{k}}{k !} \zeta(N-k)
$$

for $N<1$ and

$$
\begin{aligned}
\operatorname{Li}_{N}\left(e^{-\Delta}\right)= & \sum_{k=0, k \neq N-1}^{\infty}(-1)^{k} \frac{\Delta^{k}}{k !} \zeta(N-k) \\
& +(-1)^{N} \frac{\Delta^{N-1}}{(N-1) !}\left(\ln \Delta-H_{N-1}\right)
\end{aligned}
$$

for $N>1$; the zeta function is defined as

$$
\zeta(s) \stackrel{\operatorname{def}}{=} \sum_{m=1}^{\infty} \frac{1}{m^{s}}, \quad \operatorname{Re} s>1
$$

and $H_{N} \equiv 1+\frac{1}{2}+\frac{1}{3}+\cdots+1 / N$.

While the series (A4) is convergent for $|z|<1$, the series (A5), (A6) with the zeta function defined by Eq. (A7) seem to be divergent because the zeta argument is repeatedly equal to or smaller than 1 . This happens due to the "illegal" interchanging of the two summations. As is well known, the problem is resolved by means of the zeta function regularization procedure $[32,31]$, where the analytic continuation of $\zeta(s)$ instead of the definition (A7) is used. Then, $\zeta(1)$ remains truly divergent while

$$
\begin{gathered}
\zeta(0)=-\frac{1}{2} \\
\zeta(1-2 k)=-\frac{B_{2 k}}{2 k}, \quad k=1,2,3, \ldots \\
\zeta(-2 k)=0, \quad k=1,2,3, \ldots
\end{gathered}
$$

where $B_{l}$ are the Bernoulli polynomials.

Using Eqs. (A5), (A6) one finds that when $\Delta \rightarrow 0$

$$
\operatorname{Li}_{N}\left(e^{-\Delta}\right)=\zeta(N)+\mathcal{O}(\Delta)
$$

for $N \neq 1$. Since $\operatorname{Li}_{1}(z)=-\ln (1-z), \operatorname{Li}_{1}\left(e^{-\Delta}\right)$ diverges as $-\ln \Delta$. Therefore, when $\Delta \rightarrow 0$

$$
I_{n}^{b} \rightarrow 2(-1)^{n} T^{3-n} \zeta(3-n)
$$

for $n \neq 2$. The $n$-even contributions to the effective action vanish anyway because the respective trace of the $A$ fields equals zero; see Appendix B.

\section{Fermions}

The fermionic integrals of interest are

$$
I_{n}^{f}(a, \Delta)=T^{3-n}\left(J_{n}^{f}(a, \Delta)+(-1)^{n+1} J_{n}^{f}(-a, \Delta)\right)
$$

where $a \equiv \beta \mu$ and

$$
J_{n}^{f}(a)=\int_{\Delta}^{\infty} d x x^{2} \frac{d^{n} f_{\mathrm{FD}}(x-a)}{d x^{n}} .
$$

Expanding the Fermi-Dirac distribution as

$$
f_{\mathrm{FD}}(x-a)=\frac{1}{e^{x-a}+1}=\sum_{m=1}^{\infty}(-1)^{m-1} e^{-m(x-a)},
$$

and interchanging the order of summation and integration, we obtain after performing the integral (A2)

$$
\begin{aligned}
J_{n}^{f}(a, \Delta)= & 2(-1)^{n-1}\left(\operatorname{Li}_{3-n}\left(-e^{a-\Delta}\right)+\Delta \operatorname{Li}_{2-n}\left(-e^{a-\Delta}\right)\right. \\
& \left.+\frac{\Delta^{2}}{2} \operatorname{Li}_{1-n}\left(-e^{a-\Delta}\right)\right)
\end{aligned}
$$

Using the formula [32]

$$
\operatorname{Li}_{N}\left(-e^{-x}\right)=\sum_{n=0,}^{\infty}(-1)^{n+1} \frac{x^{n}}{n !} \eta(N-n)
$$

where $\eta(s)$ is the alternating zeta function defined as

$$
\eta(s) \stackrel{\operatorname{def}}{=} \sum_{m=1}^{\infty} \frac{(-1)^{m-1}}{m^{s}}=\left(1-2^{1-s}\right) \zeta(s), \quad \operatorname{Re} s>1
$$

we find that

$$
J_{n}^{f}(a, \Delta)=2(-1)^{n} \sum_{l=0}^{\infty} \frac{a^{l}}{l !} \eta(3-n-l)+\mathcal{O}(\Delta),
$$

when $\Delta \rightarrow 0$.

Inserting the series (A15) into Eq. (A10) one gets

$$
I_{n}^{f}(a, 0)=2 T^{3-n} \sum_{l=0}^{\infty} \frac{a^{l}}{l !} \eta(3-n-l)\left[(-1)^{n}-(-1)^{l}\right] .
$$

As seen in Eq. (A16), the argument of $\eta$ is always an even number for non-vanishing terms. Since $\eta(-2 k)=0$ for $k$ $=1,2, \ldots$ the series in Eq. (A16) terminates. Then, one observes that $I_{n}^{f}(a, 0)=0$ for $n \geqslant 4$, while

$$
\begin{aligned}
& I_{0}^{f}(a, 0)=T^{3}\left(4 a \eta(2)+\frac{2}{3} a^{3} \eta(0)\right)=T^{3}\left(\frac{\pi^{2}}{3} a+\frac{1}{3} a^{3}\right), \\
& I_{1}^{f}(a, 0)=T^{2}\left(-4 \eta(2)-2 a^{2} \eta(0)\right)=-T^{2}\left(\frac{\pi^{2}}{3}+a^{2}\right), \\
& I_{2}^{f}(a, 0)=4 a T \eta(0)=2 a T \\
& I_{3}^{f}(a, 0)=-4 \eta(0)=-2
\end{aligned}
$$

where $\eta(2)=\pi^{2} / 12$ and $\eta(0)=1 / 2$. 


\section{APPENDIX B: ADJOINT REPRESENTATION TRACES}

To compute the effective action of Eq. (5.8) and Eq. (5.9) one needs to evaluate traces in the fundamental and adjoint representations. We present here some useful formulas which relate the traces of the fundamental and adjoint representations.

First, it is easy to prove that the total symmetric traces of an odd number of adjoint generators vanish. In order to prove this, note that

$$
\operatorname{Tr}\left[\mathcal{A}_{0}^{m}\right]=\operatorname{Tr}\left[\left(\mathcal{A}_{0}^{m}\right)^{T}\right],
$$

where the superscript $T$ denotes transposition. The adjoint representation of $S U\left(N_{c}\right)$ is real, and the generators obey $T_{a}^{T}=-T_{a}$. Therefore,

$$
\operatorname{Tr}\left[\mathcal{A}_{0}^{m}\right]=(-1)^{m} \operatorname{Tr}\left[\mathcal{A}_{0}^{m}\right] .
$$

Thus, the trace vanishes for odd $m$.

Two other useful formulas are

$$
\begin{aligned}
& \operatorname{Tr} \mathcal{A}_{0}^{2}=2 N_{c} \operatorname{Tr} A_{0}^{2}, \\
& \operatorname{Tr} \mathcal{A}_{0}^{4}=6\left(\operatorname{Tr} A_{0}^{2}\right)^{2}+2 N_{c} \operatorname{Tr} A_{0}^{4} .
\end{aligned}
$$

For $N_{c}=2$ and $N_{c}=3$, one also has

$$
\operatorname{Tr} A_{0}^{4}=\frac{1}{2}\left(\operatorname{Tr} A_{0}^{2}\right)^{2}
$$

\section{APPENDIX C: THE ONE-LOOP BOSONIC ACTION}

In this appendix we show how the solutions of the transport equations allow one to reproduce the results of $[2,25,26]$. We consider only the bosonic case for the $S U(2)$ gauge group. The fermionic one can be treated similarly. Due to a global color rotation a constant background field $A_{0}$ can always be chosen in the diagonal form $A_{0}^{a}=\delta^{a 3} C / g$ where $C$ is a real constant. The respective gauge field in the adjoint representation then reads

$$
\mathcal{A}^{0}=\left(\begin{array}{ccc}
0 & -i C / g & 0 \\
i C / g & 0 & 0 \\
0 & 0 & 0
\end{array}\right) \text {. }
$$

Now, we plug this expression in Eq. (5.7) and expand the logarithm and the exponential. Taking the trace $\left[\operatorname{Tr} \mathcal{A}_{0}^{2 m}\right.$ $\left.=2(C / g)^{2 m}, \operatorname{Tr} \mathcal{A}_{0}^{2 m+1}=0\right]$, we end up with a series which after resumming reads

$$
\mathcal{L}_{g}=\frac{T}{\pi^{2}} \int_{0}^{\infty} d E E^{2}\left[\ln \left(1-e^{-\beta(E-C)}\right)+\ln \left(1-e^{-\beta(E+C)}\right)\right] .
$$

Rotating to Euclidean time $\mathcal{A}_{0} \rightarrow i \mathcal{A}_{0}^{E}$, and thus $C \rightarrow i C^{E}$, we find

$\mathcal{L}_{g}^{E}=\frac{T}{\pi^{2}} \int_{0}^{\infty} d E E^{2}\left[\ln \left(1-e^{-\beta\left(E-i C^{E}\right)}\right)+\ln \left(1-e^{-\beta\left(E+i C^{E}\right)}\right)\right]$

which matches the result found in Eq. (23) of [25]. The integral is evaluated in [25], and nonanalytic cubic terms in $C^{E}$ are found. When rotated back to the real time these terms provide imaginary contributions to the effective action. Its presence in the Minkowski effective action could have been anticipated from Eq. (C1), as for soft energies $E<C$ the logarithm becomes a multivalued complex function. The real time integral is not well-defined and an additional prescription, such as going to Euclidean time, is necessary in order to evaluate it. With the infrared cutoff $\Delta>C$, these problems are absent, and the Minkowski effective action is real.
[1] J.C. Collins and M.J. Perry, Phys. Rev. Lett. 34, 1353 (1975).

[2] D.J. Gross, R.D. Pisarski, and L.G. Yaffe, Rev. Mod. Phys. 53, 43 (1981).

[3] A.D. Linde, Phys. Lett. 96B, 289 (1980).

[4] E. Braaten and R.D. Pisarski, Nucl. Phys. B337, 569 (1990).

[5] D. Bödeker, Phys. Lett. B 426, 351 (1998); Nucl. Phys. B566, 402 (2000)

[6] S.K. Wong, Nuovo Cimento A 65, 689 (1970).

[7] U. Heinz, Phys. Rev. Lett. 51, 351 (1983); Ann. Phys. (N.Y.) 161, 48 (1985).

[8] P.F. Kelly, Q. Liu, C. Lucchesi, and C. Manuel, Phys. Rev. Lett. 72, 3461 (1994); Phys. Rev. D 50, 4209 (1994).

[9] J.C. Taylor and S.M. Wong, Nucl. Phys. B346, 115 (1990).

[10] E. Braaten and R.D. Pisarski, Phys. Rev. D 45, 1827 (1992).

[11] D.F. Litim and C. Manuel, Phys. Rev. Lett. 82, 4981 (1999); Nucl. Phys. B562, 237 (1999).

[12] P. Arnold, D.T. Son, and L.G. Yaffe, Phys. Rev. D 59, 105020 (1999).

[13] J. Jalilian-Marian, S. Jeon, R. Venugopalan, and J. Wirstam,
Phys. Rev. D 62, 045020 (2000).

[14] J. Winter, J. Phys. (Paris), Colloq. 45, C6-53 (1984).

[15] H.T. Elze, M. Gyulassy, and D. Vasak, Nucl. Phys. B276, 706 (1986).

[16] H.T. Elze, M. Gyulassy, and D. Vasak, Phys. Lett. B 177, 402 (1986).

[17] H.T. Elze, Z. Phys. C 38, 211 (1988).

[18] St. Mrówczyński, Phys. Rev. D 39, 1940 (1989).

[19] H.T. Elze and U. Heinz, Phys. Rep. 183, 81 (1989).

[20] J.P. Blaizot and E. Iancu, Phys. Rev. Lett. 70, 3376 (1993); Nucl. Phys. B417, 608 (1994).

[21] J.P. Blaizot and E. Iancu, Phys. Rep. 359, 355 (2002).

[22] J.P. Blaizot and E. Iancu, Nucl. Phys. B557, 183 (1999).

[23] D. Bödeker and M. Laine, J. High Energy Phys. 09, 029 (2001).

[24] M. Laine and C. Manuel, Phys. Rev. D 65, 077902 (2002).

[25] N. Weiss, Phys. Rev. D 24, 475 (1981).

[26] C.P. Korthals Altes, R.D. Pisarski, and A. Sinkovics, Phys. Rev. D 61, 056007 (2000). 
[27] A. Hart, M. Laine, and O. Philipsen, Nucl. Phys. B586, 443 (2000).

[28] R. Hakim, Phys. Rev. 162, 128 (1967).

[29] St. Mrówczyński and M. Thoma, Phys. Rev. D 62, 036011
(2000).

[30] H.E. Haber and H.A. Weldon, J. Math. Phys. 23, 1852 (1982).

[31] H.A. Weldon, Nucl. Phys. B270, 79 (1986).

[32] A. Actor, Nucl. Phys. B265, 689 (1986). 